Revue de l'Institut des langues et cultures

d'Europe, Amérique, Afrique, Asie et Australie

37 | 2019

Des genres en Méditerranée : pratiques,

représentations et transfert

\title{
Le merveilleux et le refoulement de la violence : une écriture névrotique du mésocosmos dans Une odeur de mantèque de Mohammed Khaïr-Eddine
}

The Wonderful and the Repression of Violence: a Neurotic Writing of the

Mesocosmos in Odeur de Mantèque of Mohammed Khair-Eddin

\section{Mohammed El Fakkoussi}

\section{OpenEdition \\ Journals}

Édition électronique

URL : http://journals.openedition.org/ilcea/7805

DOI : 10.4000/ilcea.7805

ISSN : 2101-0609

Éditeur

UGA Éditions/Université Grenoble Alpes

\section{Édition imprimée}

ISBN : 978-2-37747-099-0

ISSN : $1639-6073$

Référence électronique

Mohammed El Fakkoussi, « Le merveilleux et le refoulement de la violence : une écriture névrotique du mésocosmos dans Une odeur de mantèque de Mohammed Khaïr-Eddine », ILCEA [En ligne], 37 | 2019, mis en ligne le 04 novembre 2019, consulté le 18 janvier 2020. URL : http://journals.openedition.org/ ilcea/7805; DOI : 10.4000/ilcea.7805

Ce document a été généré automatiquement le 18 janvier 2020.

(c) ILCEA 


\section{Le merveilleux et le refoulement de la violence : une écriture névrotique du mésocosmos dans Une odeur de mantèque de Mohammed Khaïr- Eddine}

The Wonderful and the Repression of Violence: a Neurotic Writing of the

Mesocosmos in Odeur de Mantèque of Mohammed Khair-Eddin

Mohammed El Fakkoussi

1 L'imaginaire constitue un réservoir des productions conscientes et inconscientes psycho-sociales; il est un miroir où se reflète le fonctionnement archétypal de la culture. Le refoulé collectif et les images oniriques, qui nourrissent l'imaginaire d'origine, trouvent leur réplique dans les représentations socioculturelles, notamment dans l'art. Dans ce sens, l'imaginaire francophone rend compte d'une structure traditionnelle fétichiste et instaure une réflexion sur les symptômes d'ordre culturel, et après-coup sur le psychodrame d'origine.

$2 \mathrm{Au}$ demeurant, ces représentations socioculturelles sont reléguées à une sphère intermédiaire qu'Ibrahima Sow appelle le "mésocosmos ». Ce dernier « est, selon les thèmes traditionnels africains, le lieu de l'inculture, celui des doubles, des co-jumeaux "naturels" des humains.» (Sow, 1978:10) Cet espace est une réponse à des archétypes enfouis dans l'identité collective. Le corps et son double le texte seraient en communion avec les archétypes. Ceux-ci sont des schèmes embryonnaires permettant à ce corps de se réfugier dans les thèmes de la culture pattern par le truchement d'un langage tantôt délirant, tantôt poétique, là tantôt surnaturel, tantôt numineux. L'espace mésocosmique relie le refoulé collectif aux interactions sociales. Il explique en quoi une expérience psychique peut être définie selon le contexte anthropologique usuel. Dans Une odeur de mantèque, comme nous l'expliquerons plus loin, se projette le 
lyrisme paranoïaque d'une identité olfactive et visuelle orientant les trajectoires d'une identité de perception ${ }^{1}$ et fondant le récit du merveilleux ${ }^{2}$.

3 À ce titre, "la place où se situe l'expérience culturelle est l'espace potentiel entre l'individu et son environnement (originellement l'objet).» (Winnicott, 1975: 186) L'espace potentiel constitue le lieu de la vie créative où se reflète la représentation socioculturelle. Il permet de communiquer les blessures narcissiques par le transfert ${ }^{3} \mathrm{de}$ l'histoire d'une vie au moyen de l'écriture du refoulé de l'interdit. L'espace potentiel donne place à l'expérience symbolique et à ses déploiements en alimentant les thèmes de l'imaginaire socioculturel.

4 Partant de la caractérisation de cet espace potentiel, la quête identitaire chez M. KhaïrEddine est une réponse névrotique au dynamisme du mésocosmos maghrébin et une mise en narration du refoulét socioculturel sur un mode onirique. Cet espace brouille les frontières entre le moi et l'autre, commande le récit merveilleux et conditionne la mémoire d'outre-tombe d'Une odeur de mantèque.

5 Considérée comme épître des désirs ratés et du clivage identitaire, cette œuvre, écrite en 1976, est un voyage dans l'imaginaire mésocosmique, une quête délirante des signes amnésiques et une mise en scène anamnestique du corps. Car « le corps du texte vient souvent apporter au créateur un substitut du corps qui lui fait défaut » (Anzieu, 1995 : 234). L'expérience sensorielle s'inspire, d'une part, de la fiction. De l'autre, elle conditionne l'écriture, rend compte des souffrances psychosomatiques en impliquant les enjeux contextuels et reconstitue les maux du corps par les mots. Dès lors, l'expérience identitaire est représentée et reconfigurée à travers la construction du sens et la consignation épistémique de l'imaginaire d'origine.

6 L'embryon entre le réel et l'imaginaire se maintient ici par le fil de la violence subie et du refoulé inopiné. Palimpseste des odeurs répugnantes (mémoire olfactive) et chardon du déracinement, Une odeur de mantèque est une métamorphose surnaturelle d'un corps meurtri et une mémoire traumatique. L'écriture devient ainsi une esthétique de l'inachevé. Le texte ne se réduit plus à sa dimension mimétique, mais négocie son mode d'existence par le truchement de la sémiosis en ceci qu'il crée son double grâce à la résurgence du refoulé inattendu sous forme de fantasmes froids ${ }^{5}$. C'est là où l'oralité abréagit $^{6}$, à travers ses pouvoirs langagiers et sa théâtralisation, les séquelles d'une mémoire rébarbative et ancre l'écriture dans une guérilla linguistique déconstruisant le discours idéologique. Plus loin, l'entreprise de Khaïr-Eddine est d'associer le désir au sein de la mère, la névrose à une vision du monde.

De ce qui précède, nous comptons analyser, d'abord, l'espace mésocosmique où se déploie l'expérience du sujet maghrébin. Ensuite, nous dégagerons les stratégies scripturales de l'écriture névrotique, ses sublimations et ses projections comme vision du monde.

8 En effet, l'écriture constitue l'espace potentiel de l'imagination créatrice. Elle génère des liens d'échange avec le réel par le truchement du corps, sa théâtralisation et son refoulé en tant que représentation de l'imaginaire groupal. C'est ainsi que se dresse une identité subjective en interaction avec le groupe de référence et la culture-mère; identité qui s'établit par la mise en narration d'une identité narrative ${ }^{7}$, telle que la définit Paul Ricœur.

9 L'espace potentiel abrite les représentations de l'identité subjective, permet de décrypter les aléas normaux, pathologiques et sublimatoires du Moi et libère à travers 
le récit de soi le refoulé de l'imaginaire, ses moments d'ancrage et de désencrage, ses abréactions ainsi que ses transferts comme identification inconsciente au contexte socioculturel d'origine.

10 Au sein de cet espace potentiel, le récit de soi développe l'histoire d'une vie, la quête des signes identitaires par le langage du corps et ses fantasmes, la psychologisation de l'acte d'écrire la mémoire et ses refuges utopiques. C'est en cela que l'écriture identitaire ressemble selon A. Aouattah à un récit-refuge où "l'interprétation culturelle est en effet élaborée pour servir, à chaque membre de la société, [...] contre son inconscient individuel. L'avantage de cette projection de toute angoisse, profonde et intense, réside encore une fois dans la possibilité d'expulser au-dehors ce qui pourrait être ressenti comme mauvais. » (Aouattah, 2008 : 101)

11 Dans la littérature fictionnelle, comme nous le démontrerons plus loin, l'auteurnarrateur utilise l'histoire racontée comme un réservoir de scénarios dans lequel il met en narration une transposition de l'activité fantasmatique. Les maux de l'imaginaire s'évacuent à travers les mots de la trame narrative mettant en œuvre une pensée en élaboration et en lien d'identification avec un imaginaire d'origine parsemé de mauvaises graines. C'est ce qui semble expliquer l'expérience artistique, selon D. W. Winnicott, lorsqu'il situe celle-ci dans l'aire culturelle. «L'expérience culturelle, explique-t-il, commence avec le jeu et conduit à tout ce qui fait l'héritage de l'homme : les arts, les mythes historiques, la lente progression de la pensée philosophique et les mystères des mathématiques, des institutions sociales et de la religion» (1988: 50). Nous déduisons de là que la création artistique s'inscrit dans l'aire culturelle de l'espace potentiel. Cet espace s'ancre, quant à lui, dans l'espace mésocosmique où l'individu, ici le personnage, dialogue avec les fantasmes froids, refoule les blessures narcissiques et trouve refuge dans le corps-texte comme substitut du corps qui lui fait défaut.

Comme corollaire, le corps est à penser en tant que parchemin de l'expérience identitaire, qu'archive où sont mémorisées les traces indélébiles d'une mémoire rébarbative et le récit-refuge de l'imaginaire d'origine. D. Anzieu dit dans ce sens :

La fonction du Moi-peau se développe par un double appui biologique et social. Biologique: un premier dessin de la réalité s'imprime sur la peau. Social: l'appartenance d'un individu à un groupe social se marque par des incisions, scarifications, peintures, tatouages, maquillages, coiffures et leurs doublets que sont les vêtements. Le Moi-peau est un parchemin originaire, qui conserve, à la manière d'un palimpseste, les brouillons raturés, grattés, surchargés, d'une écriture « originaire » préverbale faite de traces cutanées. (1995: 128)

13 Autrement dit, le corps traduit ainsi les gribouillages de la mémoire individuelle et de celle collective, se manifeste sous forme hiéroglyphique de l'inconscient et met en narration les désirs frustrés et la violence subie à travers l'écriture du refoulé.

De ce qui précède, Une odeur de mantèque ressuscite l'histoire d'un vieillard en quête de sa terre natale. C'est un voyageur dans l'espace de la mémoire.

Il ne s'arrête jamais pour reprendre souffle, il allait devant soi, ne haletait pas, il tâchait seulement de gagner les premiers contreforts de la montagne. Il la connaissait bien, cette montagne. Sa grand-mère l'y avait souvent mené cueillir du thym et d'autres herbes dont il ignorait le nom. Il y avait même vadrouillé une fois, la nuit, pour traquer le chacal et le lièvre. Il avait alors un joli petit fusil qu'il s'était procuré comme d'habitude en le volant. Mais pourquoi s'éloigne-t-elle à présent? Était-elle toujours la même? Et lui, était-il toujours de ce monde? N'était-il pas mort et ne revivait-il pas des choses enfouies depuis longtemps, triturées par sa mémoire? (Khaï-Eddine, 2002 : 16) 

réduisant à une entité issue d'un autre monde qui se manifeste à travers des traces poussiéreuses, quitte à être comprises en tant qu'écriture originaire. d'évoquer un sentiment d'angoisse et d'inquiétude. Dans Sigmund Freud l'inquiétante étrangeté, François Stirn dit que l'inquiétante étrangeté relève d'une angoisse, voire d'une confusion de l'espace-temps. Ce qui est familier devient étrange et vice-versa. Autrement dit, "c'est ce qui n'appartient pas à la maison et pourtant y demeure " (Stirn, $2008:$ 8). Compte tenu de cette caractérisation, le récit en question met en scène la fantasmatisation d'un monde merveilleux produisant un sentiment d'inquiétante étrangeté. Le vieillard revit ses émois d'enfance et éveille les désirs ratés en les rendant rémanents.

(antôt paradisiaque, tantôt infernal. L'épreuve psychosomatique jouit ainsi d'un dynamisme scriptural. L'écriture fonctionne donc aux antipodes de la norme par une forme de liberté où se structure une réalité autrement symbolique. Le personnage restructure l'espace à travers des remparts asymétriques constitués de zones de sécurité et de menace.

Le supervieux et le vieil homme marchaient dans une clairière assourdissante, pleine de couleurs merveilleuses, d'une lumière indescriptible et de danses qu'ils ne pouvaient voir mais dont chaque battement les frôlait les remplissant d'une gaieté inédite. (C'est l'entrée du paradis cria le supervieux.) (Khaïr-Eddine, 2002 : 36-37)

C'est un monde lumineux, surchargé de couleurs, correspondant à ce qui est familier et demeurant pourtant étrange. La lumière éclaircit l'obscurité dont a été trempée la mémoire. Par-delà le délire, l'identité trouve son ordre dans cette rencontre poétique avec les éléments de la nature, notamment la lumière. Ce voyage au bout de l'être n'est entre autres que le parchemin du refoulé inopiné des désirs ratés; parchemin élaborant, de surcroît, un récit comme refuge de ce vieil homme. L'imaginaire déliriel du protagoniste est tourné vers un monde symbolique tout en frayant une échappatoire dans une terre accueillante. Son délire, pour merveilleux qu'il soit, est majestueux.

En parallèle, l'espace prend forme du moment qu'il est influencé par le sujet culturel ; c'est dire que la réalité externe tisse une relation étroite avec la réalité psychique. Cela induit une nouvelle topographie qui inclut la structure du " dehors " et du « dedans $»^{8}$. La dimension spatiale agit, dès lors, en partie sur l'état psychique de l'homme. L'expérience humaine se concrétise, dans ce sens, dans un moule intégrant une réalité spatio-temporelle. "Le monde extérieur, soutient Marcuse, est à toutes les étapes une organisation socio-historique de la réalité qui influe sur les structures mentales, par l'intermédiaire d'agences ou d'agents sociaux spécifiques » (Herbert Marcuse, 1963: 41). En effet, l'individu établit des correspondances avec le monde extérieur dans le cadre d'une expérience culturelle. La réalité psychique est ainsi conçue comme une image subjective de la dimension culturelle.

Toutefois, la perception de ce monde magique dans l'œuvre en question change brutalement et confond la notion de l'espace tout en imprégnant le récit d'une étrangeté familière. Au-delà $\mathrm{du}$ monde visible, Une odeur de mantèque crée des correspondances avec le monde invisible par le truchement d'un dynamisme à la fois symbolique et paranoïaque.

Je suis enfermé dans un cercle vicieux, se dit-il. Ce n'est pas un cercle vicieux, il devait s'en apercevoir un peu plus tard, mais un monde sans fin, une immensité 
sans pareille. [...] Brusquement, la ténèbre se dissipa, une lumière jaunâtre baignait à présent le paysage. Le vieil homme se figea, tout était sans mouvement autour de lui. La nature, si cela était bien une nature, relevait plutôt d'une énorme masse minérale. Des sons étranges l'animaient à mesure, mais rien ne paraissait s'agiter, du moins visuellement. Je ne me suis pourtant jamais drogué, se dit-il. Je n'ai jamais fumé ni mangé du haschich! Non! Je suis ici dans un autre monde, le monde des âmes mortes, des hommes dévitalisés, désincarnés. Je suis même assez loin, maintenant, du monde des djnouns et des sorciers. (Khaïr-Eddine, $2002: 32$ ) Celui-ci est pris dans une série de projections décontenancées de la réalité. Sous l'intensité du délire, cette réalité requiert une dimension dilatée dans l'espace-temps. Ainsi, l'œuvre en question exploite des techniques d'écriture qui déconstruisent le récit et embrouillent la compréhension. Même si le délire représente l'isolement et la marginalisation, il exprime, cependant, l'ouverture sur un monde symbolique. Comme le remarque Ibrahima Sow dans Les Structures anthropologiques de la folie dans l'Afrique Noire, l'espace symbolique où s'établit le lien entre l'individu et le groupe et où s'exprime en plus la représentation de la culture pattern rend intelligible l'espace d'inclusion ou d'exclusion de la personne. Cet espace témoigne, en parallèle, du fonctionnement du comportement humain qu'il soit valorisé, minorisé ou exclu, selon la pathoplasticité culturelle. De même, l'espace symbolique nourrit les formes sublimatoires qu'offre la société de référence. Sur ce plan, Ibrahima Sow constate qu'« il ne saurait y avoir de théorie ni, a fortiori, de doctrines psychiatriques valables qui ne s'élaborent à partir des lieux institutionnels et des espaces symboliques où se produisent, effectivement, l'agrégation - mais qui rendent intelligibles, aussi, les processus de désagrégation - des éléments de la personnalité » (Ibrahima sow, 1979: 7). Il en découle que la mise en narration des processus psychiques d'agrégation ou de désagrégation du personnage dans Une odeur de mantèque permet de rendre compte de l'imaginaire traditionnel en mettant en scène l'épreuve psychosomatique : le récit du rêve fournit relativement des images refoulées de la vie sociale. Il est le background des relais d'échange entre le psychisme, la contrainte sociale et la culture d'origine.

Pareillement, le délire, qui touche l'univers romanesque de l'œuvre en question, est foisonnant par ses images bestiaires.

Les crapauds qu'il [le vieux] avait crachés se tenaient en cercle autour de lui. Aussi loin que portait son regard, il ne voyait que des masses de crapauds entassés les uns sur les autres, serrées et remuants. Il se dit qu'il avait encouru un châtiment suprême pour avoir donné jour à une telle calamité, mais très rapidement il jura et convint que ce n'était là qu'une coïncidence diabolique. Quelqu'un, là-bas, avait dû lui jeter un sort. Les vieilles du village ne l'aimaient point et ne l'avaient jamais souffert parce qu'il ne leur donnait rien (Khaïr-Eddine, 2002:19).

Ainsi, de cette perception délirante de la réalité sociale, le vocabulaire de la violence et du délire emprunte ces mots de l'univers bestial. L'univers merveilleux du protagoniste est livré par conséquent à des images bestiaires. La symbolique du crapaud ${ }^{9}$ déclenche une zoopsie qui trouble la vie du vieux. C'est donc par le refoulement que le symbolisme floral et bestiaire voit le jour et inscrit le trouble psychique dans une entreprise poétique hallucinatoire et surnaturelle. "Voilà, enfin, ce que furent mes batraciens venus de l'Occident, seuls messagers possibles de ce monde déjà mort.» (Khaïr-Eddine, 2002 : 141)

Ce témoignage exprime bien le lien entre l'imagination délirante et la réalité, entre le désir hallucinatoire et la violence sociale dans une société de castration psychologique. 
Ainsi, se trouve posé, en parallèle, le rapport de la folie à l'espace-temps dont témoigne l'œuvre en question. Dans le phénomène du « jamais vu », explique Mahmoud Sami-Ali dans son article « Espace de l'inquiétante étrangeté »,

la scène la plus banale devient soudain insolite parce que, coupée de son contexte, elle se détache sur un fond vide de toute réminiscence. En revanche, la fausse reconnaissance du «déjà vu» a pour effet de dépouiller la scène de ses traits réellement énigmatiques. Perception sans souvenir ou souvenir sans perception, ce sont là deux procédés de camouflage qui visent à neutraliser le perçu en modifiant radicalement son mode d'apparition. (Sami-Ali, 1974 : 33)

Or, cette conception de l'espace imaginaire fonctionne autrement dans le mésocosmos maghrébin. Dans ce monde inidentifiable des âmes mortes se croisent le réel et l'imaginaire, le merveilleux et les fantasmes froids. C'est dans ce monde que surgissent la mémoire d'outre-tombe, la souffrance du corps et, par conséquent, la dramatisation de la violence. Celle-ci s'entend à partir d'un lieu sépulcral comme un souffle qui purge les consciences malheureuses.

Longtemps, dit le vieil homme, je voyais ces hommes vêtus de blanc s'incliner et, longtemps, je planais au-dessus d'eux en vue de faire entendre les cris de mon âme que blessaient les ailes des corbeaux et des buses. Quand je me rasseyais sur le renflement de ma tombe, l'œil, qui disait la prière des morts, dardai vers moi les racines des terres délétères, tâchant de pulvériser l'âme immortelle qui me reste (Khaïr-Eddine, $2002: 64$ ).

Le délire est tel qu'il renvoie ici à un rapport spatial et merveilleux. Il se manifeste à travers l'effacement des frontières entre le monde des hommes et celui des esprits et "opère sur une catégorie ontologique: moi / l'autre, ainsi que sur la mémoire " (Nathan, 1986:166), où le sujet confond le réel et l'imaginaire. Cette scène macabre constitue une expérience traumatique du corps qui s'étale sur une symbolique schizomorphe (délire comportemental) par une fuite ou fugue hors de ce monde. Sauf que cette fuite rencontre les réminiscences d'une mémoire blessée. Pris dans la déperdition et dans une épreuve post mortem, le corps dépeint sa déliaison avec le réel et se convertit en un délire de l'écriture.

En parallèle, dans cette épreuve morbide où le corps se trouve étranger puisqu'il ne communique avec l'autre que sur le mode du délire, le protagoniste-narrateur remémore le morcellement de ce corps qui se laisse apparaître dans la déréliction des sens, de l'espace-temps et des relations interhumaines. Ce faux-self, selon l'expression de Winnicott, reconstitue pêle-mêle l'anamnèse dont le dérèglement psychosomatique est le théâtre.

Paradoxalement, l'espace mésocosmique d'Une odeur de mantèque semble restituer un réel sans pour autant s'opposer au monde des esprits. Les associations libres ${ }^{10}$ marquent les trajectoires d'une intériorité psychique se saisissant dans un espace sépulcral. Par le truchement de ces " associations libres ", le récit rythme la réalité à sa manière et se révèle comme une contrepartie de la raison et une quête d'un monde autrement symbolique. De même, ce récit crée un monde fantasmatique et inaccessible via une imagination débordante. Aussi le corps-texte restructure-t-il un espace asymétrique à partir d'un univers symbolique.

On lava mon corps, l'ayant d'abord frotté avec des feuilles de basilic, des brindilles de thym vert et des pétales de roses, témoigne le vieil homme. Ils avaient fait bouillir l'eau du puits dans un énorme chaudron. La fumée, les escarbilles, la grande louche qu'agitait un enfant guère plus âgé que mon petit-fils, la toiture plate, la braise et les vieillards de ma connaissance qui surveillaient les gestes des laveurs, 
tout cela, je l'ai vu. Je peux même décrire la mosquée, moi déjà mort. (Khaïr-Eddine, $2002: 64-65$ )

\section{le vieil homme délire :}

Attaché à ma mère morte avant moi et enterrée dans ce cimetière. C'est peut-être elle qu'ils pleurent et c'est pour elle qu'ils amoncellent un tas d'épineux sur ma tombe: débris d'arganiers foudroyés ou laissés en plan par des bûcheronnes pressés, monceaux de jujubiers portés là par des nègres qui m'avaient taillé des sandales dans des pneus et du cuir rouge. (Khaïr-Eddine, $2002: 65$ )

31 perception se nourrit du dedans afin de reproduire, sur la base d'une identification insolite à un univers meurtrissant, le dehors comme espace spéculaire et macabre réfléchissant la violence sur un écran-archive. De cette projection émerge le refoulé inopiné et s'abréagissent les affects liés au souvenir traumatique de la mémoire culturelle : « ... ou mes enfants qui crèvent de faim en Europe, hein ? Ou ma tribu qui s'est disloquée? Ou toutes les choses que j'ai dégradées, tous les mythes qui traînent encore dans mes glaires ? [...] C'est ça que tu veux que j'éructe ?» (Khaïr-Eddine, 2002 : 117) L'interrogation permet d'actualiser la reconnaissance de soi à travers l'image de l'autre. Cette reconnaissance se maintient par le fantasme où le Moi renvoie à son propre fétiche.

Le torrent bruissait au loin, vers cette butte qu'on appelait de tous les noms, certains disaient que les djnouns y habitaient, d'autres pour qui la mante religieuse était une sorte de fétiche (la Jument des Enterrements!) gueulaient que tout cela n'était qu'un guet-apens ! [...] Tu étais non seulement ivre de toi-même mais encore prêt à te fondre dans la chair et le sang de tous ces vieux qui te racontaient des histoires et que tu savais voués à une mort prochaine... La mort n'était pour toi qu'un écran, une sorte de gêne sans plus ! (Khaïr-Eddine, 2002 : 136-137)

Si le vieil homme a le sentiment de faire partie du monde des morts, il attribue, néanmoins, son existence à son double, à savoir les histoires que racontaient les vieux. Ce clivage du personnage confère encore une fois au récit une inquiétante étrangeté, en ceci que le sujet anéanti n'a de présence qu'à travers des fantômes et des reliques.

Dans ce sens, dans l'espace de l'inquiétante étrangeté, explique Sami Ali, «le corps, en tant que sujet, a la particularité d'avoir un visage qui n'est visible que pour un autre et qui commence par être le visage de l'autre. Étant simultanément lui-même et l'autre, familier et cependant étrange, le sujet est celui qui n'a pas de visage et dont le visage se met à exister du point de vue de l'autre. » (Ali, 1974: 42-43) Après-coup, le délire se déclenche au moment de l'abolition des frontières spatio-temporelles où émergent les séquelles d'une mémoire surchargée, blessée et à jamais cicatrisée. L'évocation ou (l'éructation) de la mémoire n'est pas sans rappeler l'aliénation du vieil homme. Ce dernier "expulse au-dehors ce qui pourrait être ressenti comme mauvais", pour reprendre les mots d'Ali Aouattah. L'identification à cette mémoire blessée imprègne le récit-refuge d'une structure spatio-temporelle particulière. Ainsi, le temps devient réversible et se nourrit de l'espace mésocosmique, celui des doubles afin d'user des 
artifices permettant de sublimer la réalité traumatisante et de se réfugier dans l'espace de la mémoire collective.

Par conséquent, le merveilleux serait la zone d'échange où se cristallise l'espace de l'inquiétante étrangeté. Cela s'opère par l'incapacité de l'être à s'insérer dans l'espacetemps et par la confusion entre le fantasme et le réel. À cet égard, la déconstruction de la réalité se maintient par une distorsion du dehors qui affecte le dedans et par une intrusion inopinée de l'irrationnel dans la représentation du rationnel. La rencontre de la réalité culturelle et de celle psychique est mise en œuvre en fonction de la répétition du merveilleux, de la genèse de l'inquiétante étrangeté du réel et de l'effacement des frontières du Moi. Le sentiment de l'impassibilité de la souffrance n'est, entre autres, que ce mystérieux et monstrueux sentiment que l'individu tisse avec le réel.

Une odeur de mantèque met en narration la thématique du merveilleux comme récitrefuge d'une épreuve psychosomatique dans un espace géographique engendrant l'inquiétante étrangeté. Celle-ci implique l'espace mésocosmique où se projettent le corps anéanti, la mémoire empêchée et l'irrationnel. De là se dessine un tableau fantasmagorique, résultat d'un rapport monstrueux avec le dehors.

Dans cette atmosphère, l'espace devient le lieu d'extranéité à soi-même où la violence génère les rapports interpersonnels. Dès lors, l'individu scotomise cette réalité par le délire constant et le fantasme froid. La trame narrative se profile par la vacillation entre le merveilleux et l'espace mésocosmique.

Par conséquent, l'œuvre en question devient le réceptacle où se profilent l'esthétique, le mythe et l'impératif éthique. Par le recours à l'expérience du merveilleux, l'auteur dépeint un monde de conflits entre le moi et le surmoi via le mode onirique et par le truchement d'une représentation scripturale névrotique. Le destin du sujet en crise transpose par l'écriture le déni de la réalité socioculturelle. La dénégation de cette réalité pathogénique trouve, au niveau conscient, son expression de la forclusion, son clivage et sa perception traumatisante.

La représentation du monde ambiant tient ses fondements dans des constructions symboliques ancrées dans l'imaginaire en tant que données historiques. Ces constructions normales ou pathologiques révèlent le rapport de l'individu à son milieu socioculturel d'origine en termes de continuité dans le temps.

\section{BIBLIOGRAPHIE}

ANZIEU Didier (1995), Le Moi-peau, Paris : Éditions Dunod.

AOUATTAH Ali (2008), Interprétations et traitements traditionnels de la maladie mentale au Maroc, pour une psychiatrie « culturelle » marocaine, Casablanca : Éditions Okad.

CHEVALIER Jean \& GHEERBRANT Alain (1982), Dictionnaire des symboles, Paris : Éditions Robert Laffont. Collectif (1974), « Le Dehors et le dedans », Nouvelle Revue de psychanalyse (9), Paris : Éditions Gallimard. 
KHAÏR-EDDINE Mohammed (2002), Une odeur de mantèque, Paris : Éditions Seuil.

LAPLANCHE Jean \& PONTALIS Jean-Bertrand (1996), Vocabulaire de la psychanalyse, Paris : Éditions Delta.

MARCUSE Herbert (1963), Eros et civilisation, Paris : Les Éditions de Minuit.

NATHAN Tobie (1986), La Folie des autres, traité d'ethnopsychiatrie clinique, Paris : Éditions Dunod.

SAMI-Ali Mahmoud (1974), « Espace de l'inquiétante étrangeté », Nouvelle Revue de psychanalyse (9),

Paris : Éditions Gallimard, 33-44.

Sow Ibrahima (1978), Les Structures anthropologiques de la folie en Afrique Noire, Paris : Éditions

Payot.

STIRN François (2008), Sigmund Freud - L'inquiétante étrangeté, Grenoble : Éditions Philosophie.

WinNicotT Donald Woods (1975), Jeu et réalité, l'espace potentiel, Paris : Éditions Gallimard.

WinnicotT Donald Woods (1988), Conversations Ordinaires, Paris : Éditions Gallimard.

\section{NOTES}

1. Selon le Vocabulaire de la psychanalyse, « l'identité de perception et l'identité de pensée sont des termes employés par Freud pour désigner: ce vers quoi tendent respectivement le processus primaire et le processus secondaire. Le processus primaire vise à retrouver une perception identique à l'image de l'objet résultant de l'expérience de satisfaction. Dans le processus secondaire, l'identité recherchée est celle des pensées entre elles. " Autrement dit, dans le processus primaire, il y a une décharge immédiate; dans le processus secondaire, il y a inhibition de la satisfaction. (Jean Laplanche \& Jean-Bertrand Pontalis, 1996 :194)

2. Dans Introduction à la littérature fantastique, Tzvetan Todorov définit le merveilleux comme étant " un phénomène inconnu, encore jamais vu, à venir : donc à un futur. » (Tzvetan Todorov, Introduction à la littérature fantastique, Éditions du Seuil, Paris, 1970, p. 47)

3. «Le transfert désigne, en psychanalyse, le processus par lequel les désirs inconscients s'actualisent sur certains objets dans le cadre d'un certain type de relation établi avec eux et éminemment dans le cadre de la relation analytique. Il s'agit là d'une répétition de prototypes infantiles vécue avec un sentiment d'actualité marqué» (Jean Laplanche \& Jean-Bertrand Pontalis, Vocabulaire de la psychanalyse, op. cit., p. 492).

4. Selon Le vocabulaire de la psychanalyse, « le refoulé est l'opération par laquelle le sujet cherche à repousser ou à maintenir dans l'inconscient des représentations (pensées, images, souvenirs) liées à une pulsion. Le refoulé (refoulement) se produit dans les cas où la satisfaction d'une pulsion risquerait de provoquer du déplaisir à l'égard d'autres exigences. » (Jean Laplanche \& Jean-Bertrand Pontalis, Vocabulaire de la psychanalyse, op. cit., p. 392)

5. «Fantasme est un scénario imaginaire où le sujet est présent et qui figure, de façon plus au moins déformée par les processus défensifs, l'accomplissement d'un désir et, en dernier ressort, d'un désir inconscient. ( (Ibid., p. 152). Le fantasme froid se présente comme une identification inconsciente à une entité telle que Aicha Kandicha dans le contexte anthropologique maghrébin. 6. «Abréaction est une décharge émotionnelle par laquelle un sujet se libère de l'affect attaché au souvenir d'un événement traumatique, lui permettant ainsi de ne pas devenir ou rester pathogène. L'abréaction, qui peut être provoquée au cours de la psychothérapie, notamment sous hypnose, et produire alors un effet de catharsis, peut aussi survenir de manière spontanée, séparée du traumatisme initial par un intervalle plus ou moins long. » (Ibid., p. 1) 
7. Selon Paul Ricœur, «le récit construit l'identité du personnage, qu'on peut appeler son identité narrative, en construisant celle de l'histoire racontée. C'est l'identité de l'histoire qui fait l'identité du personnage. " (Paul Ricœur, Soi-même comme un autre, Éditions du Seuil, n 330, Paris, 1990, p. 175)

8. Ces deux termes sont utilisés en référence à la psyché (dedans) et à la culture (dehors).

9. Selon le Dictionnaire des symboles, le crapaud embrasse plusieurs symboles dans les différents imaginaires. «La peur de cet animal crépusculaire en fait communément chez nous un symbole de laideur et de maladresse [...] En Occident, le crapaud aurait été un symbole royal et solaire, antérieurement à la fleur de lys : il figure à ce titre sur l'étendard de Clovis. Mais n'y a-t-il pas, ici encore, interroge Guénon, confusion avec la grenouille, symbole de résurrection ? Le crapaud est en effet le plus souvent considéré comme l'inverse de la grenouille, dont il serait la face lunaire, infernale et ténébreuse. » (Jean chevalier \& Alain Gheerbrant, Dictionnaire des symboles, Éditions Robert Laffont, Paris, 1982, p. 309-310)

10. Associations libres : terme emprunté à l'associationnisme et désignant toute liaison entre deux ou plusieurs éléments psychiques dont la série constitue une chaîne associative. (Jean Laplanche \& Jean-Bertrand Pontalis, Vocabulaire de la psychanalyse, op.cit., p. 36)

\section{RÉSUMÉS}

L'imaginaire constitue un réservoir des productions conscientes et inconscientes psychosociales; il est un miroir où se reflète le fonctionnement archétypal de la culture. Le refoulé, les images oniriques, qui font partie de l'imaginaire d'origine, trouvent leur réplique dans les représentations socioculturelles, notamment dans l'art. Au demeurant, la production littéraire est reléguée à une sphère intermédiaire qu'Ibrahima Sow appelle le "mésocosmos ». Considérée comme épître des désirs ratés et du clivage identitaire, Une odeur de mantèque, œuvre écrite en 1976, est un voyage dans l'imaginaire de l'inculture, une quête délirante des signes amnésiques et une mise en scène du corps. L'embryon entre le réel et l'imaginaire se maintient par le fil de la perception névrotique et du refoulé inopiné. Palimpseste des odeurs répugnantes et chardon du déracinement, cette œuvre est une métamorphose surnaturelle d'un corps meurtri et une mémoire d'outre-tombe. L'écriture devient donc une esthétique de l'inachevé. Ainsi, le mythe de Khaïr-Eddine est d'associer le désir au sein de la mère, la névrose à une vision du monde.

The imaginary encompasses conscious and unconscious psychosocial productions. A mirror reflects the archetypal functioning of culture. The repressed memories and the surreal visions, which are part of the author's imaginary, are reflected in socio-cultural representations, namely in art. Literary production is, therefore, demoted to an intermediary sphere which Ibrahima Sow calls a "mésocosmos". Written in 1976, Une Odeur de mantèque, which is considered as an epistle of unfulfilled desires and identity struggles, is a journey in the traditional imaginary. It is a delusional quest for signs of amnesia and a remembrance of past representations of the body. In this book, the vital link between the real and the imaginary hangs by a thread of deranged perception and incidental repression. Being a palimpsest of unpleasant odors and a thistle (thorny issue) of deracination, this literary work is a supernatural metamorphosis of a bruised body and memory from beyond the grave. Writing, therefore, becomes an aesthetic of the 
unaccomplished. Khaïr-Eddine's myth is to associate desire with the mother's breast and neurosis with a vision of the world.

INDEX

Mots-clés : imaginaire, archétypes, mésocosmos, délire, espace potentiel, mémoire olfactive, oculaire

Keywords : the imaginary, archetypes, mésocosmos, delusion, potential space, olfactory memory, ocular

\section{AUTEUR}

MOHAMMED EL FAKKOUSSI

Université Cadi Ayyad, École Normale Supérieure, Marrakech 